\title{
Outbreaks and genetic diversity of Francisella noatunensis subsp orientalis isolated from farm-raised Nile tilapia (Oreochromis niloticus) in Brazil
}

\author{
C.A.G. Leal ${ }^{1,2}$, G.C. Tavares $^{1}$ and H.C.P. Figueiredo ${ }^{1,2}$ \\ ${ }^{1}$ AQUAVET, Laboratório de Doenças de Animais Aquáticos, \\ Departamento de Medicina Veterinária Preventiva, Escola de Veterinária, \\ Universidade Federal de Minas Gerais, Belo Horizonte, MG, Brasil \\ ${ }^{2}$ AQUACEN, Laboratório Nacional Oficial de Referência de Doenças de \\ Animais Aquáticos, Universidade Federal de Minas Gerais, \\ Ministério da Pesca e Aquicultura, Belo Horizonte, MG, Brasil \\ Corresponding author: C.A.G. Leal \\ E-mail: carlosleal@vet.ufmg.br
}

Genet. Mol. Res. 13 (3): 5704-5712 (2014)

Received May 28, 2014

Accepted June 6, 2014

Published July 25, 2014

DOI http://dx.doi.org/10.4238/2014.July.25.26

\begin{abstract}
Francisella noatunensis subsp orientalis (FNO) is an emerging pathogen of warm water tilapia in a number of different countries. The disease caused by this bacterium in fish is characterized by a systemic granulomatous infection that causes high mortality rates during outbreaks. FNO has been previously described in Asia, Europe, and Central and North America. Its occurrence in South America has never been described. Since 2012, outbreaks of a granulomatous disease have been recorded in cage farms of Nile tilapia (Oreochromis niloticus L.) in Brazil. The current study aimed to identify the etiologic agent of recent francisellosis outbreaks at Brazilian tilapia farms, and to characterize the genetic diversity of the pathogen from farms with distinct geographic origins and without epidemiological connections. Bacteriological analysis
\end{abstract}


of 44 diseased Nile tilapia collected from five cage farms in Brazil was performed during 2012 and 2013. The farms were in different locations and had no recent history of animal or biological material transport between each other. Sixty-two FNO isolates were identified on the basis of FNO-specific qPCR. The main predisposing factors for the occurrence of outbreaks on Brazilian farms were lower water temperature $\left(<22^{\circ} \mathrm{C}\right)$ and life stage of fish, affecting mainly fry, fingerlings and young adults (live weight $<100 \mathrm{~g}$ ). The genetic diversity of the Brazilian FNO isolates was evaluated using repetitive extragenic palindromic-PCR. The isolates from different origins were shown to be clonally related. This is the first report of the occurrence and genetic diversity of FNO in South America.

Key words: Clonality; Francisellosis; Tilapia; Predisposing factors; REP-PCR

\section{INTRODUCTION}

Francisella noatunensis is an important emerging pathogen responsible for outbreaks of francisellosis at fish farms in a number of different countries. The subspecies, F. noatunensis subsp noatunensis and F. noatunensis subsp orientalis (FNO; syn. Francisella asiatica), have been shown to be associated with francisellosis in the chicken grunt (Parapristipoma trilineatum), hybrid striped bass (Morone saxatilis x M. chrysops), Atlantic salmon (Salmo salar), Atlantic cod (Gadus morhua), tilapia (Oreochromis spp), ornamental cichlid, and damselfish (Chromis viridis) (Hsieh et al., 2007; Birkbeck et al., 2011; Camus et al., 2013). Francisellosis primarily affects the fry and fingerling stages of fish, which exhibit systemic granulomatosis with multifocal granulomas in the internal organs, including the liver, spleen, and kidney (Soto et al., 2009, 2012a).

Cases of francisellosis caused by FNO in farm-raised tilapia have been reported in Taiwan (Chen et al., 1994), Costa Rica (Soto et al., 2009), Indonesia (Ottem et al., 2009), the UK (Jeffery et al., 2010), and the USA (Soto et al., 2011). However, data regarding FNO infections at fish farms in South America are scant. In Brazil, outbreaks of granulomatous disease in Nile tilapia (Oreochromis niloticus L.) fingerlings reared in floating cages have been reported since 2012. Although the etiology of these cases was unclear, the clinical signs and field presentation of the disease were suggestive of francisellosis.

The aims of current study were to identify the etiologic agent of the recent outbreaks of francisellosis in farm-raised Nile tilapia in Brazil, and to characterize the genetic diversity of isolates from different farms on the basis of genomic fingerprinting.

\section{MATERIAL AND METHODS}

\section{Outbreaks}

During the winter months of 2012 and 2013, we investigated outbreaks of granulomatous disease with high mortality at five Nile tilapia farms in distinct geographic regions of the Brazilian State of Minas Gerais. The fish at these farms were reared in floating cages, and no epidemiological connection existed between the farms. The disease occurred when the water temperature was $22^{\circ} \mathrm{C}$ or less. We collected a total of 44 diseased fingerlings and juveniles. 
The fish were transported to the laboratory on ice, and the bacteriological analysis was performed immediately upon arrival.

\section{Bacteriology}

For bacterial isolation, aseptically obtained kidney and spleen swabs from each fish were streaked onto cysteine heart agar supplemented with $2 \%$ bovine hemoglobin $(\mathrm{CHAH}$; BD Biosciences, USA). The plates were incubated at $28^{\circ} \mathrm{C}$ for 4 to 7 days. Isolates were subjected to Gram staining, and were tested for catalase and oxidase activity. The isolates were stored at $-80^{\circ} \mathrm{C}$ in Mueller-Hinton cation-adjusted broth supplemented with $2 \%$ VX supplement (Laborclin, Brazil), 0.1\% glucose (MMH), and 15\% glycerol.

\section{DNA extraction and 16S rRNA gene (16S rDNA) sequencing}

The isolates were thawed, streaked onto $\mathrm{CHAH}$ and incubated at $28^{\circ} \mathrm{C}$ for $48-72 \mathrm{~h}$. Total DNA was extracted from ten randomly selected isolates using the DNeasy Tissue \& Blood kit (Qiagen, Germany), according to manufacturer instructions. The amount of extracted DNA was quantified using a NanoDrop spectrophotometer (Thermo Scientific, Germany). Amplification and sequencing of the 16S rRNA gene were performed for all 12 isolates. 16S rRNA was amplified by PCR with the universal primers C70 (5'-AGAGTTTGATYMTGGC-3') and B37 (5'-TACGGYTACCTTGTTACGA-3') according to the method described by Fox et al. (1995). PCR products were purified using a Wizard PCR Preps kit (Promega, USA) and sequenced using forward and reverse primers. Sequencing reactions were performed using a BigDye ${ }^{\mathrm{TM}}$ Terminator Cycle sequencing kit and run on an ABI 3730XL genetic analyzer (both from Life Technologies, USA). The 16S rDNA sequences were compared with those of reference strains in GenBank (http://ncbi.nlm.nih.gov) using the BLASTn computational tool, and nucleotide sequence identity $\geq 98 \%$ was used as the criterion for species identification.

\section{Real-time PCR (qPCR) identification}

The identity of all of the isolates was confirmed using FNO-specific qPCR, as previously described (Soto et al., 2010). The primers and probe were obtained from Sigma-Aldrich (USA). The 5' and 3' termini of the hydrolysis probe were labeled with 6-carboxy-fluorescein and the non-fluorescent Black Hole Quencher 1, respectively. The qPCR assays were performed using the TaqMan Universal Master Mix (Life Technologies) in a ViiA 7 Real-Time PCR System (Life Technologies). The data acquisition and analysis were performed using the ViiA 7 software. The qPCR data were evaluated according to the guidelines established by Bustin et al. (2009) regarding the minimum information for the publication of quantitative real-time PCR experiments.

\section{Genetic diversity}

The genetic diversity of the isolates was evaluated using repetitive extragenic palindromic-PCR (REP-PCR), as previously described (Ouoba et al., 2010). PCR was performed using the HotStart Taq Polymerase kit (Qiagen). The PCR samples contained 1X PCR buffer, 
$0.5 \mu \mathrm{M}$ GTG5 primer (5'-GTGGTGGTGGTGGTG-3'), $0.2 \mu \mathrm{M}$ dNTPs, 2 mM MgCl 2,2 U Taq DNA polymerase, and $60 \mathrm{ng}$ template DNA. Thermal cycling was performed in a Verity 96Well Thermal Cycler (Life Technologies) using an initial denaturation step at $95^{\circ} \mathrm{C}$ for $15 \mathrm{~min}$, followed by 30 cycles of $95^{\circ} \mathrm{C}$ for $30 \mathrm{~s}, 45^{\circ} \mathrm{C}$ for $1 \mathrm{~min}$, and $72^{\circ} \mathrm{C}$ for $4 \mathrm{~min}$, with a final elongation at $72^{\circ} \mathrm{C}$ for $8 \mathrm{~min}$. The primer was obtained from Life Technologies. The PCR products were separated by electrophoresis on a $1.5 \%$ agarose gel, and stained with $0.5 \mu \mathrm{g} / \mathrm{mL}$ ethidium bromide for $30 \mathrm{~min}$. The gel images were captured using the L-Pix EX digital imaging system (Loccus Biotecnologia, Brazil), and the images were analyzed using the BioNumerics, version 6.6, software (Applied Maths, Belgium). The Dice coefficient was used to analyze the similarities in the banding patterns (Dice, 1945). The discriminatory power of each typing method was calculated using the Simpson diversity index $(D)$ (Hunter and Gaston, 1988). Dendrograms were constructed using the unweighted pair group average method. Isolates that shared sequence similarity $\geq 80 \%$ were considered to be clonally related (Soto et al., 2012b).

\section{Experimental infection}

To fulfill Kock's postulate, Nile tilapia fingerlings were experimentally infected with a FNO strain isolated from diseased fish. The strain FNO-12 was randomly selected for challenge assay. The protocol for our in vivo experiment was approved by the Ethics Committee in Animal Experimentation of Universidade Federal de Minas Gerais (Brazil). We obtained 50 Nile tilapia fingerlings with an average weight of $45 \mathrm{~g}$ from a commercial hatchery. The fish were divided into four experimental groups, with ten fish in each group. The groups were maintained in separate 57-L tanks supplied with flow-through dechlorinated tap water $(0.5 \mathrm{~L} / \mathrm{h})$ at a water temperature of $26^{\circ} \mathrm{C}$. Fish were fed to satiation four times a day with commercially available feed containing 32\% crude protein (Presence, Brazil). Ten fish were randomly selected for bacteriological analysis and FNO-specific qPCR to ensure that they were free of FNO infection.

The bacterial suspension was then adjusted to an optical density corresponding to approximately $10^{6} \mathrm{CFU} / \mathrm{mL}$. The fish were anesthetized by immersion in a bath containing $10 \mu \mathrm{g} / \mathrm{mL}$ benzocaine, and a $0.1-\mathrm{mL}$ bacterial inoculum was administered by intraperitoneal injection, corresponding to a final dose of $10^{6} \mathrm{CFU} /$ fish. The control group was injected intraperitoneally with $0.1 \mathrm{~mL}$ sterile $\mathrm{MMH}$. After inoculation, challenged fish were monitored for 15 days four times a day. Samples of kidney and spleen tissues were collected from all dead fish for re-isolation of bacteria. The re-isolates were identified by FNO-specific qPCR.

\section{RESULTS}

\section{Outbreak characterization and bacteriology}

During the winter months of 2012 and 2013, five outbreaks of granulomatous disease with high mortality occurred at different commercial Nile tilapia farms in Minas Gerais, Brazil. The farms were located at different, geographically separated dams. The outbreaks occurred when water temperatures were $\leq 22^{\circ} \mathrm{C}$. There was no recent history of animal transport between any of the farms, and the farms acquired fry from different suppliers. The field presentation and pathogenesis of the disease was similar at all farms. Death was preceded by a 5- to 7-day period of anorexia. The major clinical signs were melanosis, exophthalmia, erratic swimming, skin 
ulcers (primarily at the base of the fins), and gill pallor. At necropsy, the principal pathological features were nephromegaly, splenomegaly, and hepatomegaly, all of which were accompanied by multifocal white nodules. The histological examination of the nodules indicated granulomatous lesions (data not shown). Fingerlings, juveniles, and young adults were affected. The body weight of diseased fish ranged from 2.04 to $109.3 \mathrm{~g}$. Mortality ranged from 30 to $60 \%$. On the basis of these observations, the major predisposing factors were water temperature $\leq 22^{\circ} \mathrm{C}$ and the fingerling, juvenile, and young adult life stages. Mortality at these farms substantially decreased, or was almost reduced to $0 \%$ when the water temperature rose to $\geq 24^{\circ} \mathrm{C}$.

Sixty-two isolates were obtained from the 44 diseased fish collected (Table 1). The BLASTn analysis of the 16S rDNA sequences of the Brazilian FNO isolates showed $99 \%$ sequence similarity with the 16S rDNA of LADL-07-285A (GenBank accession number CP006875.1) and Toba 04 (NR_074581.1) strains of FNO. The identity of all 62 FNO isolates was confirmed by the FNO-specific qPCR analysis.

\begin{tabular}{|c|c|c|c|c|c|}
\hline Strain & Farm & Year & Strain & Farm & Year \\
\hline FNO 01-12 & A & 2012 & FNO 43-13 & D & 2013 \\
\hline FNO 02-12 & A & 2012 & FNO 44-13 & D & 2013 \\
\hline FNO 03-12 & A & 2012 & FNO 45-13 & D & 2013 \\
\hline FNO 04-12 & A & 2012 & FNO 46-13 & D & 2013 \\
\hline FNO 05-12 & A & 2012 & FNO $47-13$ & $\mathrm{D}$ & 2013 \\
\hline FNO 06-12 & A & 2012 & FNO 48-13 & D & 2013 \\
\hline FNO 07-12 & A & 2012 & FNO 49-13 & $\mathrm{D}$ & 2013 \\
\hline FNO 08-12 & A & 2012 & FNO $50-13$ & D & 2013 \\
\hline FNO 09-12 & A & 2012 & FNO $51-13$ & D & 2013 \\
\hline FNO 10-12 & A & 2012 & FNO $52-13$ & D & 2013 \\
\hline FNO 11-12 & A & 2012 & FNO 53-13 & D & 2013 \\
\hline FNO 12-12 & A & 2012 & FNO 54-13 & D & 2013 \\
\hline FNO 13-12 & A & 2012 & FNO 55-13 & D & 2013 \\
\hline FNO 14-12 & A & 2012 & FNO 56-13 & D & 2013 \\
\hline FNO 15-12 & A & 2012 & FNO $57-13$ & D & 2013 \\
\hline FNO 16-12 & A & 2012 & FNO 58-13 & D & 2013 \\
\hline FNO 17-12 & A & 2012 & FNO 59-13 & D & 2013 \\
\hline FNO 18-12 & A & 2012 & FNO 60-13 & D & 2013 \\
\hline FNO 19-12 & A & 2012 & FNO 61-13 & E & 2013 \\
\hline FNO 20-12 & B & 2012 & FNO 62-13 & E & 2013 \\
\hline FNO 21-12 & B & 2012 & & & \\
\hline FNO 22-12 & B & 2012 & & & \\
\hline FNO 23-12 & B & 2012 & & & \\
\hline FNO 24-12 & B & 2012 & & & \\
\hline FNO 25-12 & B & 2012 & & & \\
\hline FNO 26-12 & B & 2012 & & & \\
\hline FNO 27-12 & B & 2012 & & & \\
\hline FNO 28-12 & B & 2012 & & & \\
\hline FNO 29-12 & B & 2012 & & & \\
\hline FNO 30-12 & B & 2012 & & & \\
\hline FNO 31-12 & B & 2012 & & & \\
\hline FNO 32-12 & B & 2012 & & & \\
\hline FNO 33-12 & B & 2012 & & & \\
\hline FNO 34-12 & B & 2012 & & & \\
\hline FNO 35-12 & B & 2012 & & & \\
\hline FNO 36-12 & B & 2012 & & & \\
\hline FNO 37-12 & $\mathrm{C}$ & 2012 & & & \\
\hline FNO 38-12 & $\mathrm{C}$ & 2012 & & & \\
\hline FNO 39-12 & $\mathrm{C}$ & 2012 & & & \\
\hline FNO 40-12 & $\mathrm{C}$ & 2012 & & & \\
\hline FNO 41-13 & $\mathrm{C}$ & 2013 & & & \\
\hline FNO 42-13 & $\mathrm{C}$ & 2013 & & & \\
\hline
\end{tabular}




\section{Genetic diversity and challenge assay}

REP-PCR resulted in the amplification of eleven bands, ranging in size from 300 to $1850 \mathrm{bp}$. Seven different REP patterns were obtained with a discriminatory power of 0.639 . However, on the basis of the similarity threshold of $80 \%$, the 62 isolates were shown to be clonally related (Figure 1).

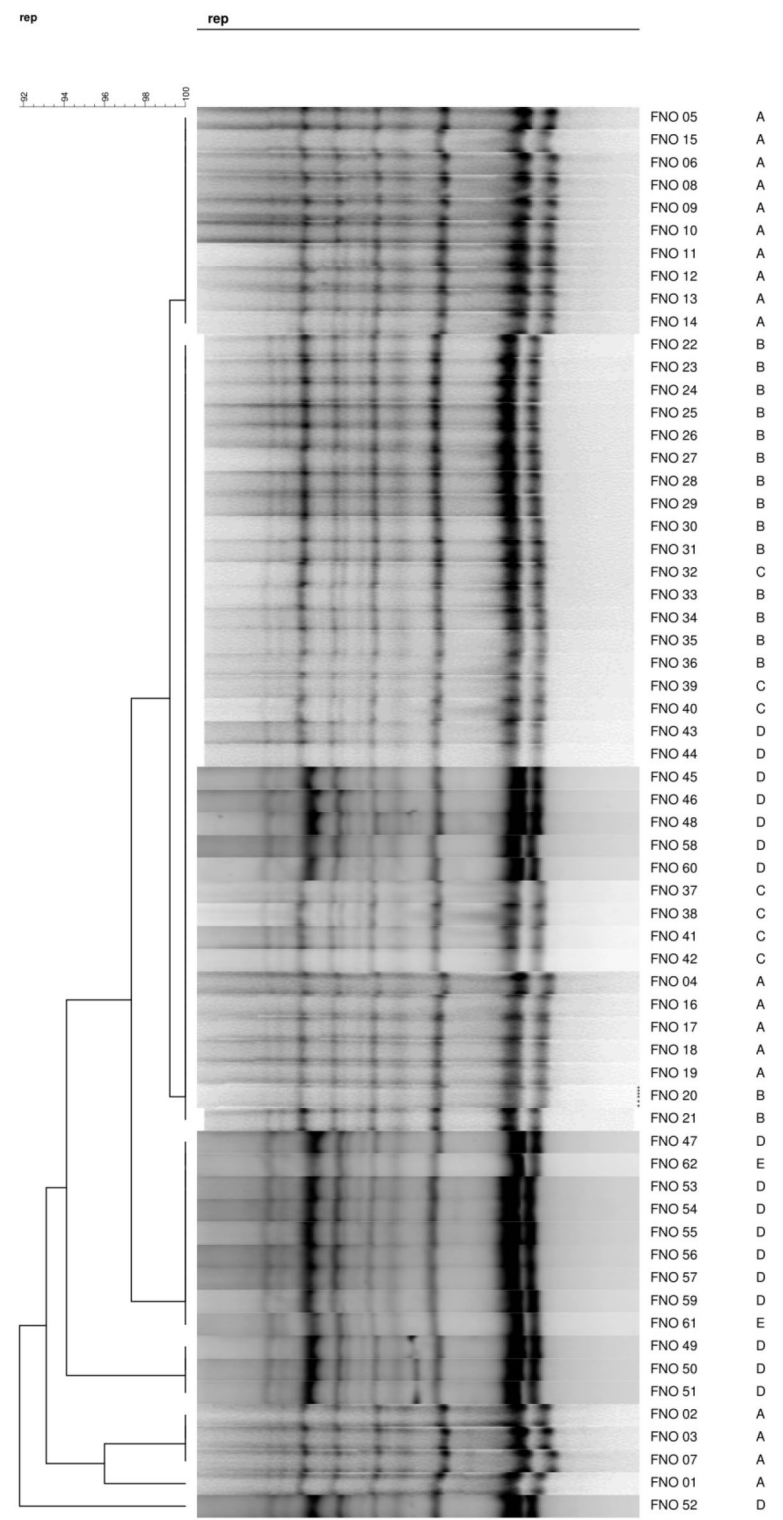

Figure 1. REP-PCR-based genotyping analysis of 62 isolates of Francisella noatunensis subsp orientalis from Nile tilapia in Brazil. The dendrogram was constructed using Dice's coefficient and the UPGMA method. 
The mortality rate in the experimental infection was $90 \%$ for challenged groups. After bacterial challenge with the FNO-12 isolate, the major clinical signs first appeared at $6 \mathrm{~h}$ postinoculation in two challenged groups, and included anorexia, lethargy, melanosis, exophthalmia, and ascites. The first mortality was recorded at $20 \mathrm{~h}$ post-inoculation in trial $1\left(26^{\circ} \mathrm{C}\right)$. The last mortality was observed $8 \mathrm{~h}$ post-inoculation. In the analysis of the fish that died during the observation period, the necropsy examination revealed splenomegaly, hepatomegaly, renal congestion, and the presence of multifocal white nodules in the spleen, kidney, and liver. The bacterium was re-isolated from the kidney and spleen from all dead fish. All isolates showed positive results in FNO-specific qPCR. The two surviving fish at the end of trial were euthanized and subjected to bacteriological examination. These remaining fish at the end of experimental period showed positive results in bacteriology for FNO. The species of the strains re-isolated was confirmed by FNO-specific qPCR.

\section{DISCUSSION}

Since 2012, outbreaks of a granulomatous disease at Nile tilapia farms in Minas Gerais and other southeastern Brazilian states have been reported. These outbreaks occurred during the winter, and affected mainly cage-reared fingerlings and juveniles. We investigated five outbreaks in geographically separated Nile tilapia floating cage farms in 2012 and 2013. FNO was identified as the etiologic agent in all cases examined. The diseased fish presented with anorexia, melanosis, exophthalmia, erratic swimming, skin ulcers, and gill pallor. These clinical signs were similar to previously described outbreaks of FNO-related francisellosis in tilapia in Central America (Mauel et al., 2007; Soto et al., 2009), Taiwan (Chen et al., 1994), Indonesia (Ottem et al., 2009), the UK (Jeffery et al., 2010), and the USA (Soto et al., 2011). Our findings represent the first report of outbreaks of FNO-related francisellosis in South America.

The FNO infections at the Brazilian Nile tilapia farms resulted in high mortality rates. At some of the farms, mortality reached $60 \%$ of the total fish stocks. Previous reports of outbreaks at tilapia farms in other regions of the world did not present data for total mortality based on verified cases (Soto et al., 2009, 2011; Jeffery et al., 2010). Mortality rates of up to $40 \%$ in Atlantic cod have been reported for francisellosis caused by F. noatunensis subsp noatunensis (Colquhoun and Duodu, 2011), which is lower than that caused by FNO.

The major predisposing factors for the francisellosis outbreaks at the Nile tilapia cage farms in Brazil were the life stage and water temperature. Our results are consistent with those of previous studies, which showed that Nile tilapia fingerlings are the life stage most susceptible to FNO infection (Jeffery et al., 2010; Soto et al., 2011, 2013). In addition, the occurrence of disease in young adult tilapia ( $>100 \mathrm{~g}$ ) was confirmed. Soto et al. (2013) also reported FNO infection in 100-g Nile tilapia reared in indoor aquaculture facilities in the USA.

Water temperature is a key factor in $\mathrm{FNO}$ outbreaks at tilapia farms. In a previous study, fish reared at a water temperature of $25^{\circ} \mathrm{C}$ developed francisellosis that resulted in considerably higher mortality than that observed in those reared at $30^{\circ} \mathrm{C}$ (Soto et al., 2012c). Mauel et al. (2003) also reported higher mortality among tilapia reared at an average water temperature of $24.45^{\circ} \mathrm{C}$, compared with that observed among fish reared at $26.96^{\circ} \mathrm{C}$. A recent study of Mozambique tilapia (Oreochromis mossambicus) in Hawaii showed that the incidence of FNO infection was higher when the water temperature was $25^{\circ} \mathrm{C}$ or less (Soto et al., 2013). Outbreaks of francisellosis in Nile tilapia reared indoors have occurred at water 
temperatures ranging from $24^{\circ}$ to $28^{\circ} \mathrm{C}$ (Jeffery et al., 2011; Soto et al., 2011). By contrast, the francisellosis outbreaks in Brazil occurred when the water temperature was $22^{\circ} \mathrm{C}$ or lower. The conditions in the floating cages (mainly high stock densities and intensive husbandry) of the farms in Brazil and water temperatures below $22^{\circ} \mathrm{C}$ could suppress the immune response in fish. This issue associated with high virulence of Brazilian FNO strains could explain the high mortalities found during outbreaks.

The genetic structure of FNO populations worldwide has not been well characterized. Soto et al. (2012b) evaluated the genetic diversity of 10 strains of FNO isolated from different fish species in two different countries using ERIC-PCR and BOX-PCR, and all were found to be clonally related. We obtained similar results for the Brazilian FNO strains. The 62 isolates were identified as FNO using qPCR, and we genotyped them using REP-PCR. Despite some minor variation in REP-PCR patterns, all 62 isolates were found to be clonally related. In both studies, FNO isolates showed a high degree of homogeneity, suggesting a clonal genetic structure of this bacterium population in each case. However, the genetic relationships between FNO isolates from Taiwan, Costa Rica, Indonesia, the UK, the USA, and Brazil remain unclear. Future studies of the genetic relationships between FNO isolates from different countries are warranted.

\section{CONCLUSION}

This is the first report of francisellosis outbreaks caused by FNO in South America. The genotyping results revealed that the Brazilian isolates comprised a highly homogeneous population.

\section{ACKNOWLEDGMENTS}

Research supported by the Ministry of Fisheries and Aquaculture and the National Center for Animal Information-INCT/CNPq/UFMG. The authors wish to thank FAPEMIG, CAPES, and CNPq for awarding a student fellowship for this project.

\section{REFERENCES}

Birkbeck TH, Feist SW and Verner-Jeffreys DW (2011). Francisella infections in fish and shellfish. J. Fish. Dis. 34: 173-187.

Bustin SA, Benes V, Garson JA, Hellemans J, et al. (2009). The MIQE guidelines: minimum information for publication of quantitative real-time PCR experiments. Clin. Chem. 55: 611-622.

Camus AC, Dill JA, McDermott AJ, Clauss TM, et al. (2013). Francisella noatunensis subsp. orientalis infection in IndoPacific reef fish entering the United States through the ornamental fish trade. J. Fish. Dis. 36: 681-684.

Chen SC, Tung MC, Chen SP, Tsai JF, et al. (1994). Systematic granulomas caused by a rickettsia-like organism in Nile tilapia, Oreochronuis niloticus (L.), from southern Taiwan. J. Fish. Dis. 17: 591-599.

Colquhoun DJ and Duodu S (2011). Francisella infections in farmed and wild aquatic organisms. Vet. Res. 42: 47.

Dice LR (1945). Measures of the amount of ecologic association between species. Ecology 26: 297-302.

Fox JG, Yan LL, Dewhirst FE, Paster BJ, et al. (1995). Helicobacter bilis sp. nov., a novel Helicobacter species isolated from bile, livers, and intestines of aged, inbred mice. J. Clin. Microbiol. 33: 445-454.

Hsieh CY, Wu ZB, Tung MC and Tsai SS (2007). PCR and in situ hybridization for the detection and localization of a new pathogen Francisella-like bacterium (FLB) in ornamental cichlids. Dis. Aquat. Organ. 75: 29-36.

Hunter PR and Gaston MA (1988). Numerical index of the discriminatory ability of typing systems: an application of Simpson's index of diversity. J. Clin. Microbiol. 26: 2465-2466. 
Jeffery KR, Stone D, Feist SW and Verner-Jeffreys DW (2010). An outbreak of disease caused by Francisella sp. in Nile tilapia Oreochromis niloticus at a recirculation fish farm in the UK. Dis. Aquat. Organ. 91: 161-165.

Mauel MJ, Miller DL, Frazier K, Liggett AD, et al. (2003). Characterization of a piscirickettsiosis-like disease in Hawaiian tilapia. Dis. Aquat. Organ. 53: 249-255.

Mauel MJ, Soto E, Moralis JA and Hawke J (2007). A piscirickettsiosis-like syndrome in cultured Nile tilapia in Latin America with Francisella spp. as the pathogenic agent. J. Aquat. Anim. Health 19: 27-34.

Ottem KF, Nylund A, Karlsbakk E, Friis-Møller A, et al. (2009). Elevation of Francisella philomiragia subsp. noatunensis Mikalsen et al. (2007) to Francisella noatunensis comb. nov. [syn. Francisella piscicida Ottem et al. (2008) syn. nov.] and characterization of Francisella noatunensis subsp. orientalis subsp. nov., two important fish pathogens. $J$. Appl. Microbiol. 106: 1231-1243.

Ouoba LI, Nyanga-Koumou CA, Parkouda C, Sawadogo H, et al. (2010). Genotypic diversity of lactic acid bacteria isolated from African traditional alkaline-fermented foods. J. Appl. Microbiol. 108: 2019-2029.

Soto E, Hawke JP, Fernandez D and Morales JA (2009). Francisella sp., an emerging pathogen of tilapia, Oreochromis niloticus (L.), in Costa Rica. J. Fish. Dis. 32: 713-722.

Soto E, Bowles K, Fernandez D and Hawke JP (2010). Development of a real-time PCR assay for identification and quantification of the fish pathogen Francisella noatunensis subsp. orientalis. Dis. Aquat. Organ. 89: 199-207.

Soto E, Baumgartner W, Wiles J and Hawke JP (2011). Francisella asiatica as the causative agent of piscine francisellosis in cultured tilapia (Oreochromis sp.) in the United States. J. Vet. Diagn. Invest. 23: 821-825.

Soto E, Illanes O, Hilchie D, Morales JA, et al. (2012a). Molecular and immunohistochemical diagnosis of Francisella noatunensis subsp. orientalis from formalin-fixed, paraffin-embedded tissues. J. Vet. Diagn. Invest. 24: 840-845.

Soto E, Griffin M, Wiles J and Hawke JP (2012b). Genetic analysis and antimicrobial susceptibility of Francisella noatunensis subsp. orientalis (syn. F. asiatica) isolates from fish. Vet. Microbiol. 154: 407-412.

Soto E, Abrams SB and Revan F (2012c). Effects of temperature and salt concentration on Francisella noatunensis subsp. orientalis infections in Nile tilapia Oreochromis niloticus. Dis. Aquat. Organ. 101: 217-223.

Soto E, McGovern-Hopkins K, Klinger-Bowen R, Fox BK, et al. (2013). Prevalence of Francisella noatunensis subsp. orientalis in cultured tilapia on the island of Oahu, Hawaii. J. Aquat. Anim. Health 25: 104-109. 